\title{
Chromosome Studies in Indian Pandanales
}

\author{
Ranajit Mallick and Arun Kumar Sharma \\ Cytogenetics Laboratory, Department of Botany, University of Calcutta, \\ 35, Ballygunj Circular Road, Calcutta 19, India
}

Received November 2, 1965

\section{Introduction}

The systematic positions of the three monocotyledonous genera Pandanus, Typha and Sparganium have been much debated. Though the three have been placed in different families by all taxonomists, the principal controversy involves their affinities. Engler and Prantl (1930) included all the three families under one order Pandanales in their system of classification. Hutchinson (1959), on the other hand, separated Typhaceae and Sparganiaceae under the order Typhales and Pandanaceae under Pandanales. Moreover in the latter system the two orders are widely separated from one another.

In addition to the positions of the three genera the status of Pandanales in evolution is also a matter of controversy. Radically different views are held by Engler and Prantl on one hand and Hutchinson on the other. The simplicity of the flower, that is unisexual and naked with anemophilous method of pollination, led Engler and Prantl to consider Pandanales as the most primitive order of the monccotyledons. As such it has been placed at the beginning of the series. These authors consider that evolution is progressive and increase in complexity in structure is the principal feature in evolution.

Hutchinson considered regression as one of the principal features in evolution and regarded that decrease in complexity should not be ignored in the study of evolution. According to him the simplicity of Pandanales is not due to primitiveness but due to reduction and extreme specialisation of apparently simple forms. According to him, bisexual flowers with large number of floral organs, specially the stamens and carpels and apocarpous gynoecium, are evidences of primitiveness. On that basis he considered the orders of Helobiae as the starting point of monocotyledons. Pandanales were not only split but assigned an advanced status.

Lately, the importance of cytology in solving problems of taxonomic dispute has been well-recognised. From a comparative study of number and morphology of the chromosomes including the karyotypic details as well as the meiotic behaviour where necessary, it is not only possible to trace the affinities between different taxa but also to determine their phylogenetic status. Some of the generalisations which are considered as criteria for this deduction are well known. Low number and long size of the chromosomes and the symmetrical karyotype are considered as distinct evidences of primitiveness. Gradual asymmetry, associated with polyploidy and diminution in chromatin matter, represent evolutionary advance. How far all these criteria can be applied to Pandanales in solving its taxonomic controversy is worth consideration.

In spite of the taxonomic problem associated with Pandanales no detailed cytological work has yet been carried out on any of the three genera excepting the stray reports of mere chromosome numbers of a few of the species. Therefore the present investigation was undertaken with the object of settling the taxonomic dispute in relation to these taxa, with the aid of cytological studies. 


\section{Materials and methods}

Materials: The present investigation includes seven species belonging to the three genera under the three families of the order Pandanales. A list of the names, identified from the Indian Botanic Garden, Shibpur is given below:

1. Pandanus odoratissimus Linn.

2. P. furcatus Roxb.

3. P. sanderi Hort.

4. P. utilis Bory.

5. Typha angustifolia Linn.

6. T. elephantina Roxb.

7. Sparganium ramosum Huds.

The genera Pandanus and Typha are more or less tropical and subtropical and they are distributed generally throughout the world but the distribution of Sparganium is very limited. The genus Sparganium with very few species is distributed mainly in the temperate regions of the Western India (Kashmir) and some of the islands of the Eastern hemisphere (Java, Sumatra, etc.).

The plants, for the present study, are mainly procured from the local nurseries and were also collected from the suburbs of Calcutta and the only one species of Sparganium that is available in India was collected from the temperate regions of the Kashmir Valley.

Methods: For the study of somatic chromosomes a great deal of difficulty was encountered due to their semi-aquatic and marshy habit, karyotypic studies were mainly made from the temporary preparations, as in the permanent preparations with different metallic and nonmetallic fixatives, they were not as clear as the temporary ones.

In temporary squash preparations, different pretreating chemicals were tried and the best results were obtained in .01 M solution of 8-oxyquinoline for 3 hours (Tjio and Levan 1950 ) and saturated solution of paradichlorobenzene for 5 hours (Sharma and Mookerjea 1955). The root tips for the sake of better penetration of the pretreating fluid were first suctioned through a suction pump and then kept in the above chemicals at a very low temperature $\left(2^{\circ}-4^{\circ} \mathrm{C}\right)$ for $5-10$ mins. After that they were transferred to a comparatively higher temperature $\left(14^{\circ}-16^{\circ} \mathrm{C}\right)$ and kept there for two hours and forty-five mins. in case of oxyquinoline and four hours and forty-five mins. in case of paradichlorobenzene. For obtaining uniform results $.01 \mathrm{M}$ oxyquinoline was generally used for pretreatment.

After pretreatment, the root tips were fixed in acetic alcohol $(1: 1)$ for 1 hour, heated in a mixture of $2 \%$ aceto-orcein and $(\mathrm{N}) \mathrm{HCl}(9: 1)$ solution for a few seconds. Some crystals of ferric acetate were also added in the mixture to intensify the staining. The roots were kept there for $24 \mathrm{hrs}$. and then squashed in $45 \%$ acetic acid. But as the cytoplasm takes heavy stain and chromosome configuration is not well clarified so the roots after fixation were hydrolysed in $\mathrm{NHCl}$ for $10-12$ mins. at $60^{\circ} \mathrm{C}$ and stained in basic fuchsin or they were hydrolysed in $\mathrm{NHCl}$ for $30-60$ secs., stained in $2 \%$ ace to basic fuchsin solution (basic fuchsin dissolved in $40 \%$ acetic acid) and squashed in $30 \%$ acetic acid.

Permanent preparations of the temporary squash were made by inverting the slides in tertiary butyl alcohol till the cover slips detached off and then mounted in euparal.

For meiotic study temporary squash preparations were made following Belling's 1\% aceto-carmine technique.

The figures were drawn at a table magnification of $\times 2900$ using a Zeiss microscope with a compensating eye-piece of $\times 20$, an apochromatic objective of 1.3 N.A. and an aplanatic condenser of 1.2 N.A.

In the drawings and idiograms, the chromosomes with secondary constrictions or satellites have been drawn only in outlines. 


\section{Observation}

The members of the order Pandanales show a remarkable constancy in the chromosome number. The different species of Pandanus show $2 \mathrm{n}=60$, whereas the two species of Typha and one species of Sparganium, so far investigated, show $2 \mathrm{n}=30$ chromosomes. The chromosomes are extremely small and without practically any size variation. The chromosomes with secondary constrictions are comparatively longer than the others. The variation in chromosome number is also not noticeable.

The gross homogeneity in the karyotypes is not only evident at the specific level but also at the generic level. The meiotic study, where made, also showed regular bivalent formation.

A general description of the different types depending on the locations of primary and secondary constrictions is given below:

Type A: Represented by comparatively long chromosomes, each with two distinct constrictions, primary and secondary, one submedian in position and the other a satellite at the distal end of the short arm.

Type B: Represented by comparatively long chromosomes, each with a submedian primary constriction and a satellite at the distal end of the long arm.

Type C: Consists of medium-sized chromosomes each with a median primary constriction and a satellite at one arm.

Type D: Is represented by comparatively long chromosomes with submedian primary constrictions.

Type E: Consists of short chromosomes with median primary constrictions.

Family: Pandanaceae Genus: Pandanus

Four species have been worked out.

1. Pandanus odoratissimus Linn. $\left(2 \mathrm{n}=60=\mathrm{A}_{2}+\mathrm{B}_{2}+\mathrm{C}_{2}+\mathrm{E}_{54}\right)$

Sixty chromosomes have been observed in the normal somatic complement of the species. The chromosomes are very small and their size ranges between $1.03 \mu$ to $1.7 \mu$. Three pairs of chromosomes, A, B and C, bear secondary constrictions (Figs. 1 and 12a).

2. Pandanus furcatus Roxb. $\left(2 \mathrm{n}=60=\mathrm{A}_{4}+\mathrm{B}_{2}+\mathrm{C}_{2}+\mathrm{D}_{2}+\mathrm{E}_{50}\right)$

The normal somatic complement of the species bears sixty chromosomes. The chromosomes are very small and their size ranges between $1.02 \mu$ to $1.8 \mu$. Three pairs of chromosomes, A, B and C, bear secondary constrictions. The types of chromosomes are not sharply differentiated, one type merging with the other (Figs. 2 and 12b).

3. Pandanus sanderi Hort. $\left(2 \mathrm{n}=60=\mathrm{B}_{2}+\mathrm{C}_{2}+\mathrm{E}_{\mathrm{\varepsilon} 6}\right)$

The normal somatic complement of the species possesses sixty chromo- 
somes. The chromosomes are very small and their size ranges between $1.03 \mu$ to $1.7 \mu$. The pairs of chromosomes $\mathrm{B}$ and $\mathrm{C}$ bear secondary constrictions (Figs. 3 and 12c).
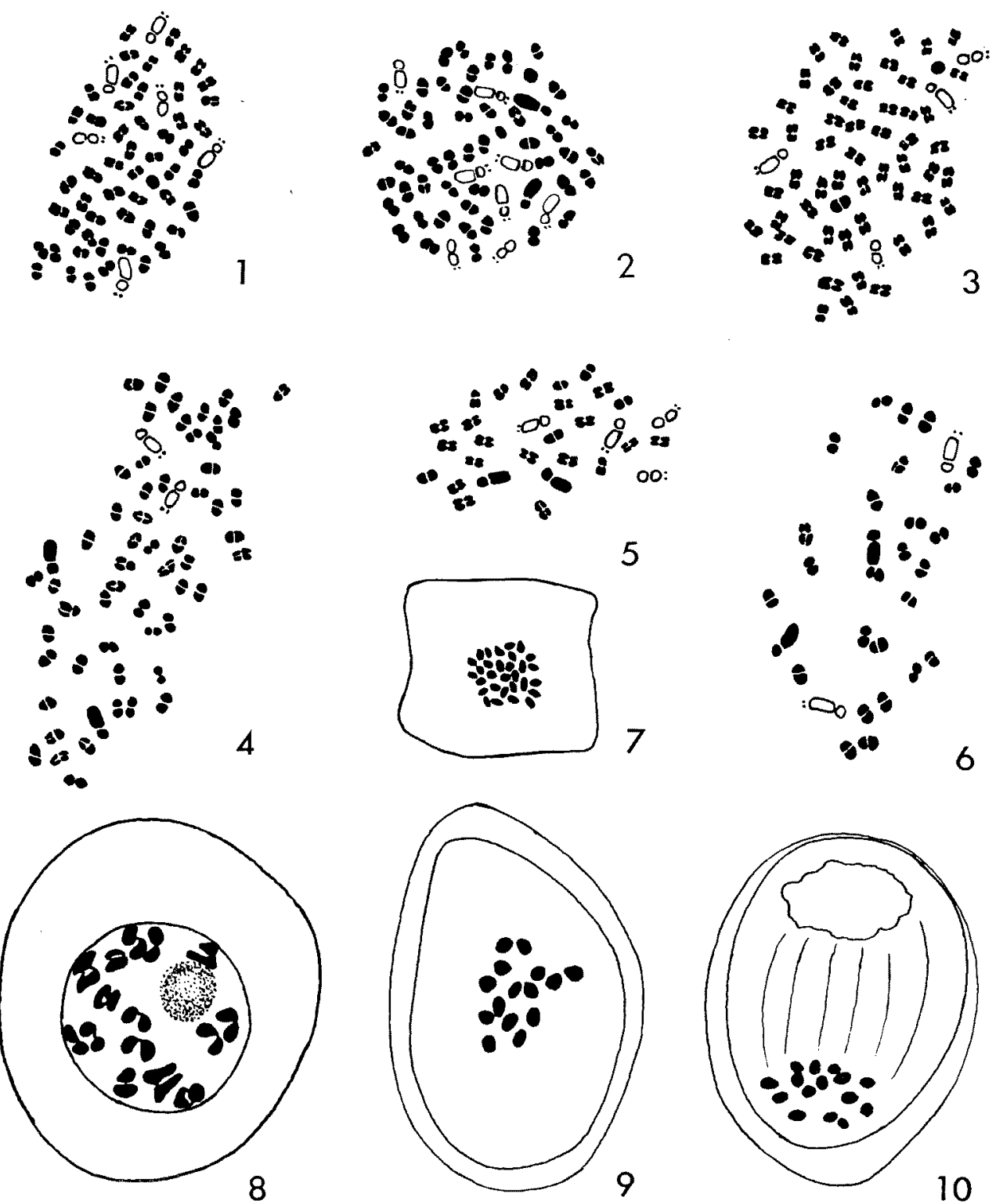

Figs. 1-10. 1, Pandanus odoratissimus, somatic metaphase with $2 \mathrm{n}=60$ chromosomes. $2, P$. furcatus, somatic metaphase with $2 \mathrm{n}=60$ chromosomes. $3, P$. sanderi, somatic metaphase with $2 \mathrm{n}=60$ chromosomes. $4, P$. utilis, somatic metaphase with $2 \mathrm{n}=60$ chromosomes. 5, Typha angustifolia, somatic metaphase with $2 \mathrm{n}=30$ chromosomes. 6 , T. elephantina, somatic metaphase with $2 \mathrm{n}=30$ chromosomes. 7 , Sparganium ramosum, somatic metaphase with $2 \mathrm{n}=30$ chromosomes. 8-10, S. ramosum, meiotic stages showing 15 bivalents in diakinesis, metaphase $\mathrm{I}$ and anaphase $\mathrm{I}$.

4. Pandanus utilis Bory. $\left(2 \mathrm{n}=60=\mathrm{B}_{3}+\mathrm{D}_{2}+\mathrm{E}_{56}\right)$

The somatic complement of the species normally contains sixty chromo- 
somes. The chromosomes are in general very small. Their size ranges between $1.02 \mu$ to $1.7 \mu$. One pair of chromosomes B bears secondary constriction (Figs. 4 and 12d).

Family: Typhaceae Genus: Typha

Two species have been worked out.

1. Typha angustifolia Linn. $\left(2 \mathrm{n}=30=\mathrm{B}_{2}+\mathrm{C}_{2}+\mathrm{D}_{2}+\mathrm{E}_{24}\right)$

The somatic complement of the species consists of thirty chromosomes. The chromosomes are very small and their size ranges between $1.02 \mu$ to $1.7 \mu$. Two pairs of chromosomes, B and $\mathrm{C}$, bear secondary constrictions (Figs. 5 and 12e).

2. Typha elephantina Roxb. $\left(2 \mathrm{n}=30=\mathrm{B}_{2}+\mathrm{D}_{2}+\mathrm{E}_{20}\right)$

Thirty chromosomes have been found to be present in the normal somatic complement of the species. The chromosome size is very small and ranges between $1.03 \mu$ to $1.7 \mu$. One pair of chromosomes, B, bears secondary constrictions (Figs. 6 and 25).

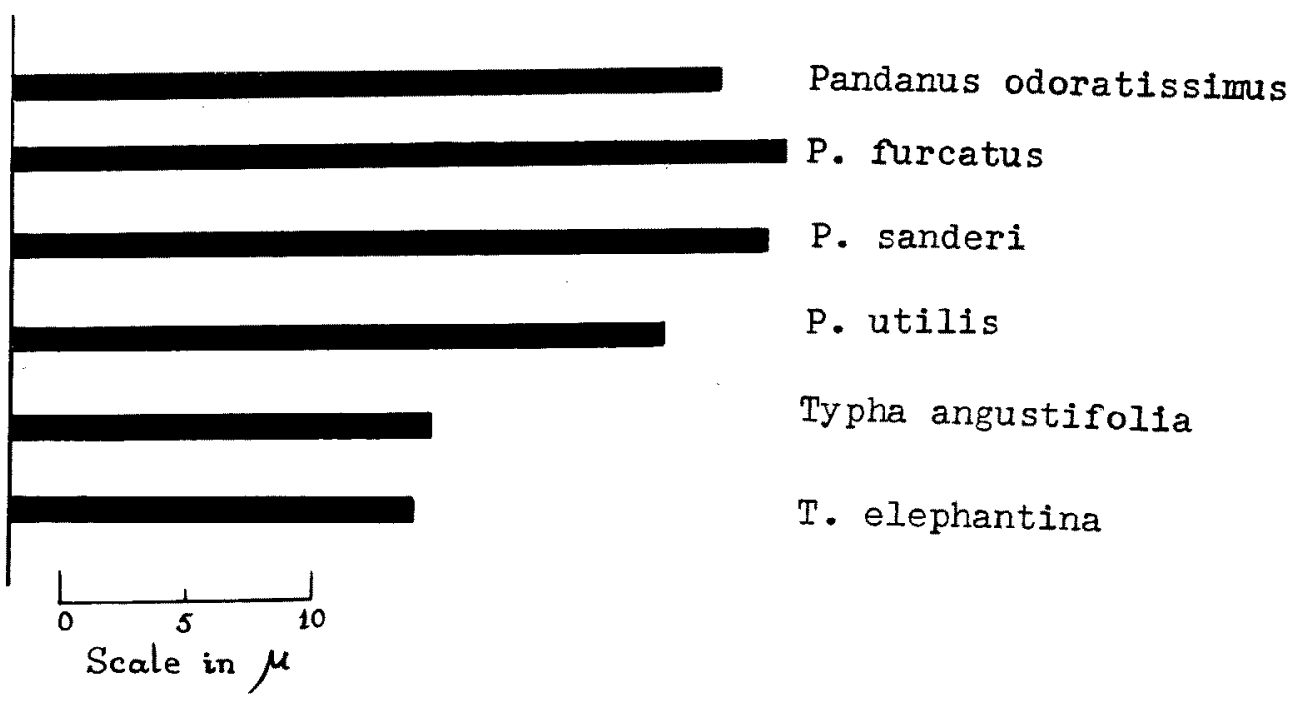

Fig. 11. Histogram showing the total amount of chromatin matter of the different species of Pandanales.

Family: Sparganiaceae Genus: Sparganium

1. Sparganium ramosum Huds.

Meiotic study of only one available species of Indian Sparganium, S. ramosum, has been worked out. It shows fifteen clear bivalents in diakinesis (Fig. 8). Metaphase I shows fifteen bivalents (Fig. 9) and the anaphase I also shows regular separation of fifteen bivalents (Fig. 10). No irregularity in meiosis is observed.

Due to the non-availability of the material, somatic study could not be made and so the somatic figure has been reproduced here from the paper of 
H. D. Wulff in Ber. Deutsch. Bot. Gesellschaft. Bd. LIV (Tafel IV), 1938. The figure shows $2 \mathrm{n}=30$ chromosomes (Fig. 7).

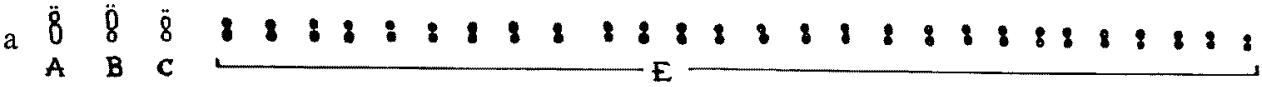

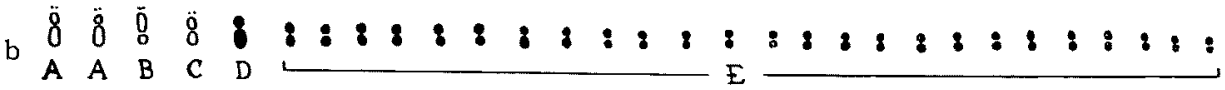

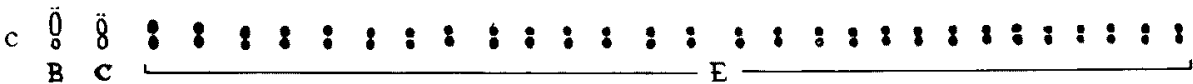

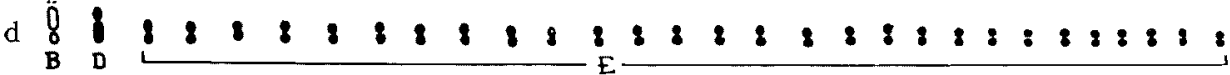

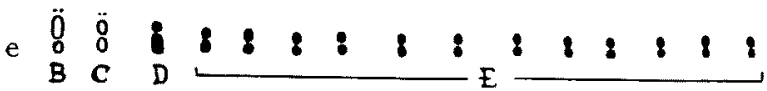

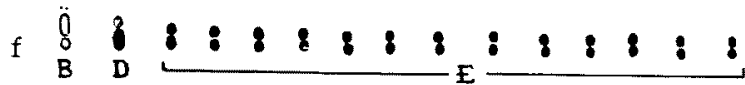

Fig. 12. Idiograms of Pandanus and Typha. a, P. odoralissimus. b, P. furcatus. c, P. sanderi. d. $P$. utilis. e. T. angustifolia. f. T. elephantina.

\section{Discussion}

1. Chromosome characteristics of the three genera

The different species of Pandanus investigated here are all characterised by a common chromosome number of $2 \mathrm{n}=60$. Tjio (1948) studied two species of Pandanus, viz. P. pacificus and P. pygmaeus in both of which $2 \mathrm{n}=60$ chromosomes were recorded. In P. boninensis, Harada (1949) observed $2 \mathrm{n}=64$ chromosomes, a report which should not be relied upon as Harada himself reported the number as an approximate count. It is quite clear therefore that $2 n=60$ characterises all species of Pandanus as evidenced from the previous and present reports.

In addition to the uniformity in chromosome number of different species, the general similarity in morphology is easily detectable as revealed from the study of their karyotypes. Short chromosomes characterise the chromosome complement of all the species with slight size difference in all the complement. On the comparatively longer chromosomes the satellites are located. The number of satellites and the position of the constrictions in the comparatively longer chromosomes vary from species to species, suggesting that minor differences in the karyotype might have been an associated feature related to speciation. Constrictions in general vary from median to submedian in position. The number and morphology of the chromosomes indicate clearly that the genus Pandanus represents a natural assemblage. The evolution 
of new species might have been influenced by minor karyotypic alterations noted in them.

The two other genera Typha and Sparganium have also revealed quite interesting cytological result. Both the species of Typha which are available in India show $2 \mathrm{n}=30$ chromosomes. Previous record too reveals $2 \mathrm{n}=30$ chromosomes in four species of Typha including the two present ones (Löve and Löve 1942, Harada 1949). In both the species the chromosome morphology shows marked similarity with slight difference in the number of satellited chromosomes. Therefore the genus Typha too, as far as the evidences go, shows the possibility of being a natural assemblage. However, detailed karyotype study is needed for other species as well, growing in different countries.

So far as Sparganium is concerned only one species, $S$. ramosum is available in India. Meiotic studies in here reveal clear 15 bivalents. Wulff (1938) has carried out somatic studies in this species. All the other species of Sparganium worked out by previous authors (Löve and Löve 1942, 1948, Harada 1949) show in all cases $2 \mathrm{n}=30$, and in S. stenophyllum (Harada 1949), in addition, a triploid individual have been recorded. The chromosome number is quite uniform in Sparganium too.

If all the three genera Pandanus, Typha and Sparganium are considered together, remarkable affinity between them in their cytology can be easily detected. In Typha and Sparganium the basic number is $\mathrm{x}=15$ whereas in Pandanus it is $\mathrm{x}=30$, just the multiple of the former. In addition to the chromosome number the karyotype similarity is remarkable. A glance at the idiogram reveals that it is very difficult to distinguish between the chromosome complements of Pandanus and Typha. The different chromosome types that have been observed in Pandanus are present in Typha as well. The similar type of gross morphology is observable in the figure presented by Wulff (1938), in Sparganium ramosum. All these factors taken together indicate the close affinity of all the four genera.

2. An assessment of taxonomic status in the light of the present investigations

Engler and Prantl, in their system of classification, not only included these three families under Pandanales but suggested that Sparganium is most related to Pandanus specially in the character of the inflorescence, occasional union of carpel and in the method of apparent dichotomy in the branches

Hutchinson, who separated Pandanales from Typhales, the latter including Sparganiaceae and Typhaceae, contended that both have evolved from a Liliaceous stock. Typhales is directly derived from this stock whereas Pandanales owes an indirect origin through Agavales and Palmales. His principal reason for separating the two orders is based on the criteria of habitat, habit and also the structure of the inflorescence. Typhales of Hutchinson is represented by aquatic and marsh habit with elongate linear leaves sheathing 
at the base. Pandanales on the other hand are trees and shrubs with stilt root, with leaves spirally arranged and inflorescence with spathe like bracts. Typhaceae, having a naked flower with one to many stamens and carpels, which led Engler and Prantl to include all the three genera under one order, has been completely ignored by Hutchinson. Special emphasis is laid on habit and habitat in his system.

The cytological studies as presented here reveal remarkable affinities between the three genera and indicate that their wide separation, as suggested by Hutchinson, seems to be quite unnatural. The retention under one order claimed by Engler and Prantl appears to be quite justified (cf. Harada 1949).

With regard to the evolutionary status of Pandanales, cytological studies however provide no support to Engler's contention of its primitiveness. The chromosome number is fairly high, much higher than that of Helobiae. At the same time short size of the chromosomes indicates their advanced status. Therefore from the external morphological characteristics, taken in conjunction with the cytological data, Huthinson's contention of the advanced status of Pandanales appears to be fairly justified. It may be suggested that the proper arrangement would be to include these genera under three different families under one order Pandanales-an order representing quite an advanced level in evolution, which might have been derived from the Liliaceous stock as suggested by Hutchinson.

\section{Summary}

The present work deals with the cytology and karyotype analysis of the seven species of the order Pandanales belonging to the three genera-Pandanus, Typha and Sparganium under three distinct families. The karyotypic studies show $2 \mathrm{n}=60$ chromosomes in case of Pandanus and $2 \mathrm{n}=30$ chromosomes in case of Typha and Sparganium. Meiotic studies in only one species of Sparganium show regular 15 bivalents.

The uniformity in chromosome number with extremely small size is the characteristic features of the three genera. In Sparganium and Typha the chromosome number is $2 n=30$, whereas in Pandanus it is $2 n=60$. The chromosome morphology of the different species of the genera shows slight differences in their structure particularly in the number of satellited chromosomes.

Cytological studies show the close affinities of the three genera thus supporting Engler and Prantl's contention. However they also indicate their advanced status, thus supporting Hutchinson's view.

\section{References}

Darlington, C. D. and Wylie, A. P. 1955. Chromosomes Atlas of Fowering Plants. George Allen and Unwin, Ltd., London. 
Engler; A. and Prantl, K. 1930. Die Natürlichen Pflazenfamilien. Berlin, 15a.

Harada, I. 1949. Chromosome numbers in Pandanus, Sparganium and Typha. Cytologia 14: $214-218$.

Hutchinson, J. 1959. Families of Flowering Plants. II. Monocotyledons. MacMillan \& Co. Ltd., London.

Löve, A. and Löve, D. 1942. Chromosome numbers of Scandinavian plant species. Bot. Not. 1942: 19-59.

- 1948. Chromosome numbers of Northern plant species. Icel. Univ. Inst. Appl. Sci. Dept. Agric. Rep. B 3: 1-131.

Sharma, A. K. and Mookerjea, A. 1955. Paradichlorobenzene and other chemicals in chro. mosome work. Stain Tech. 30: 1-7.

Tjio, J. H. 1948. The Somatic Chromosomes of Some Tropical Plants. Hereditas 34: 135.

- and Levan, A. 1950. The use of oxyquinoline in chromosome analysis. Ann. Expt. Aula-Dei 2: 61-64.

Wulff, H. D. 1938. Chromosomenstudien an der Schleswigholsteinischen Angiospermen. Flora 3. Ber. Der. Deuts. Ges. 44: 